\title{
Endovascular Cerebral Venous Sinus Imaging with Optical Coherence Tomography
}

\author{
(D)C.R. Pasarikovski, (D).C. Ku, (D). Keith, (D). Ramjist, (D). Dobashi, (D) S.M. Priola, (D) L. da Costa, and (D).X.D. Yang
}

\begin{abstract}
BACKGROUND AND PURPOSE: Imaging of the cerebral venous sinuses has evolved Substantially during the past 2 decades, and most recently intravascular sinus imaging with sonography has shed light on the pathophysiology of sinus thrombosis and intracranial hypertension. Optical coherence tomography is the highest resolution intravascular imaging technique available but has not been previously used in cerebral sinus imaging. The purpose of this study was to develop a preclinical animal model of endovascular optical coherence tomography cerebral venous sinus imaging and compare optical coherence tomography findings with histology.
\end{abstract}

MATERIALS AND METHODS: Four consecutive Yorkshire swine were selected. The superior sagittal sinus was first catheterized with a microwire, and the optical coherence tomography catheter was delivered via a monorail technique into the sinus. Luminal blood was cleared with a single arterial injection. After structural and Doppler optical coherence tomography imaging, a craniotomy was performed and the sinus and adjacent dura/veins were resected. Bland-Altman analysis was performed to compare optical coherence tomography and histology.

RESULTS: Technically successful optical coherence tomography images were obtained in 3 of 4 swine. The luminal environment and visualization of dural arteries and draining cortical veins were characterized. The average maximum diameters of the sinus, dural arteries, and cortical veins were $3.14 \mathrm{~mm}, 135 \mu \mathrm{m}$, and $260 \mu \mathrm{m}$, respectively. Bland-Altman analysis demonstrated good agreement between histology and optical coherence tomography images.

CONCLUSIONS: Endovascular optical coherence tomography imaging was feasible in this preclinical animal study. Adoption of this imaging technique in the human cerebral venous sinus could aid in the diagnosis, treatment, and understanding of the pathophysiology of various diseases of the sinus. Human safety and feasibility studies are needed.

ABBREVIATIONS: $\mathrm{CVS}=$ cerebral venous sinuses; $\mathrm{dOCT}=$ Doppler OCT; IIH = idiopathic intracranial hypertension; IVUS = intravascular ultrasound; $\mathrm{OCT}=$ optical coherence tomography

$\mathrm{T}$ he cerebral venous sinuses (CVS) are rigid structures located between the periosteal and meningeal layers of the dura matter. ${ }^{1}$ The sinuses act as reservoirs for collecting venous blood from both the deep and superficial venous systems of the brain and are lined with endothelium and elastic lamina. ${ }^{2}$ They lack the

Received April 14, 2020; accepted after revision July 28.

From the Division of Neurosurgery (C.R.P., J.C.K.), Department of Surgery, University of Toronto, Toronto, Ontario, Canada; Department of Anatomic Pathology (J.K.), Sunnybrook Health Sciences Center, Toronto, Ontario, Canada; Division of Neurosurgery (J.R., Y.D., L.d.C., V.X.D.Y.), Sunnybrook Hospital, University of Toronto, Toronto, Ontario, Canada; Division of Neurosurgery (S.M.P.), Department of Surgery, Health Sciences North, Sudbury, Ontario, Canada; and Hurvitz Brain Sciences Research Program (V.X.D.Y.), Sunnybrook Research Institute, Sunnybrook Health Sciences Centre, University of Toronto, Toronto, Ontario, Canada.

Please address correspondence to Christopher R. Pasarikovski, MD, SurgeonScientist Training Program, Division of Neurosurgery, University of Toronto, 399 Bathurst St, WW 4-427 Toronto, ON M5T 2S8 Canada; e-mail:

chris.pasarikovski@mail.utoronto.ca

http://dx.doi.org/10.3174/ajnr.A6909 smooth-muscle layers found in most blood vessels and have no valves. ${ }^{3}$ Diseases of the CVS include dural arteriovenous fistulas, cerebral venous sinus thrombosis, and idiopathic intracranial hypertension (IIH).

Imaging of the CVS has evolved considerably during the past 2 decades. CTV and MRV, with either 2D-TOF or contrastenhanced MRV, are the current imaging modalities of choice for the diagnosis of cerebral venous sinus thrombosis or venous sinus stenosis in $\mathrm{IIH}^{4}{ }^{4}$ However, large studies comparing the sensitivity and specificity of MRV for determining the degree of stenosis with DSA, which is the presumed criterion standard for cerebral venous imaging, are lacking. Boddu et $\mathrm{al}^{5}$ showed that contrastenhanced MRV significantly overestimated the size of sinus stenosis in patients with $\mathrm{IIH}$ compared with intravascular ultrasound (IVUS), concluding that contrast-enhanced MRV would be a poor technique for stent-size selection. Similarly, the accuracy of DSA has been questioned owing to the limitations of $2 \mathrm{D}$ 


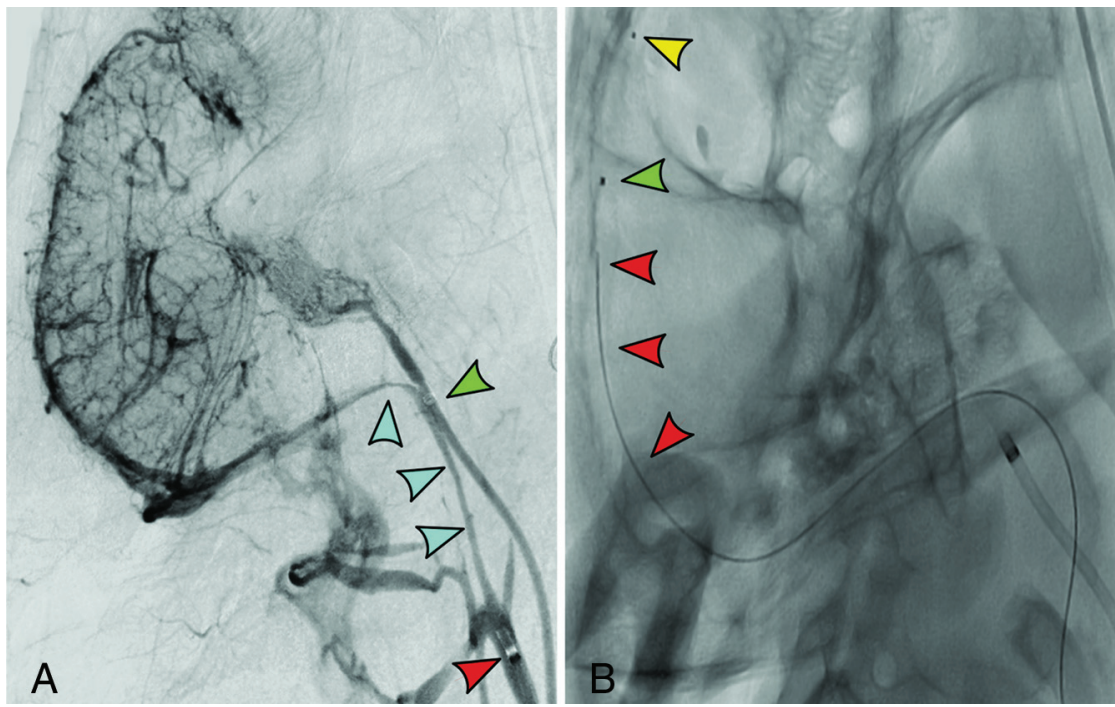

FIG 1. Swine cerebral venous sinus. A, Combined arterial and venous diagnostic angiography injections in a lateral view via a guide catheter tip in the ascending pharyngeal artery (green arrow) and the internal jugular vein (red arrow), revealing the connection between the internal jugular vein and sigmoid sinus (blue arrows). B, OCT catheter in the superior sagittal sinus. The distal catheter marker (yellow arrow), lens marker (green arrow), and optical fiber (red arrows) are visible in the superior sagittal sinus.
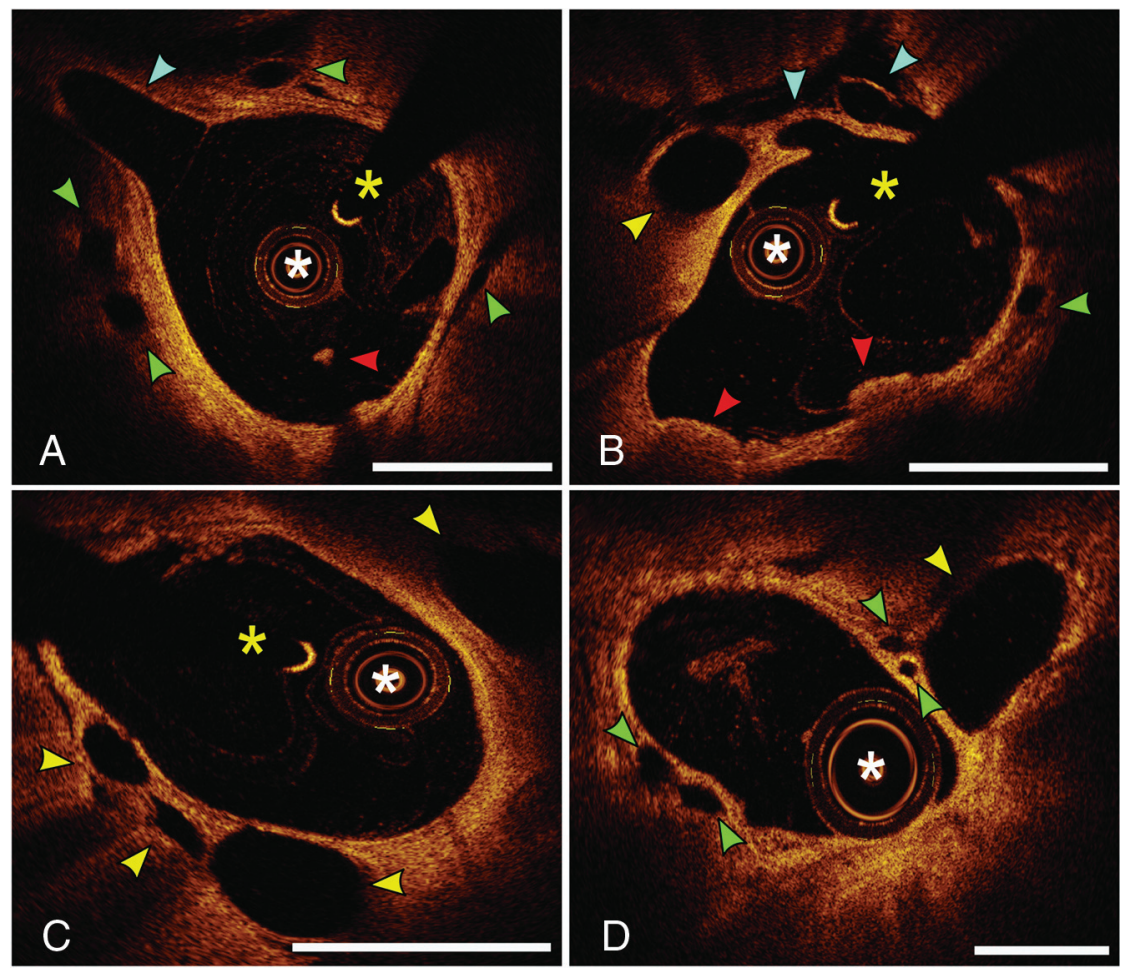

FIG 2. Cross-sectional OCT imaging within the superior sagittal sinus. Large draining cortical veins can be observed entering the sinus (light blue arrow) with multiple adjacent dural arteries visible (green arrows), along with adjacent cortical veins outside the sinus lumen (yellow arrows). Small red thrombi (red arrows) are also visible in certain sections, either free-floating or attached to the sinus wall. The white asterisk is the OCT lens, and the yellow asterisk is the artifact from the wire. White bars $=2 \mathrm{~mm}$. planar imaging. Karmon et $\mathrm{al}^{6}$ compared DSA with IVUS and reported that angiography was less sensitive in describing the luminal environment, frequently missing luminal thrombus, valves in the internal jugular vein, flaps, and septations.

Endovascular optical coherence tomography (OCT) is the highest resolution intravascular imaging technique available, using near-infrared light with a wavelength of approximately $1300 \mathrm{~nm}$, and excellent spatial resolution of $10 \mu \mathrm{m}$ is achievable. ${ }^{7}$ In comparison, IVUS has a spatial resolution of $100 \mu \mathrm{m}$ (approximately 10 times less resolution), and $3 \mathrm{~T}$ MR vessel wall imaging has a voxel size of $2.0 \times 0.4 \times 0.4 \mathrm{~mm}$. With near-histologic resolution, OCT has been described as an optical biopsy technique. ${ }^{8}$

We hypothesized that endovascular OCT would enable superior characterization of the cerebral sinus luminal environment and would visualize draining cortical veins and dural arteries. To our knowledge, endovascular OCT imaging of the human CVS has not been undertaken. The purpose of this research was to develop a proof of concept animal endovascular OCT cerebral venous sinus imaging model and compare OCT imaging with histology. Doppler OCT images were also acquired.

\section{MATERIALS AND METHODS}

All experiments were conducted according to the policies and standards established by the authors' institutional animal research ethics board (Sunnybrook Health Sciences Center Research Ethics Board). Four consecutive Yorkshire swine weighing $40-45 \mathrm{~kg}$ were used for sinus imaging. There was no prescreening imaging of venous anatomy for any animal. All procedures were performed with the animal under a general anesthetic with continuous hemodynamic monitoring. The animals were fed standard diets at our facility for 2 weeks before the procedure.

Ultrasound-guided right femoral punctures were performed, and a $6 \mathrm{~F}$ sheath was inserted into the right 

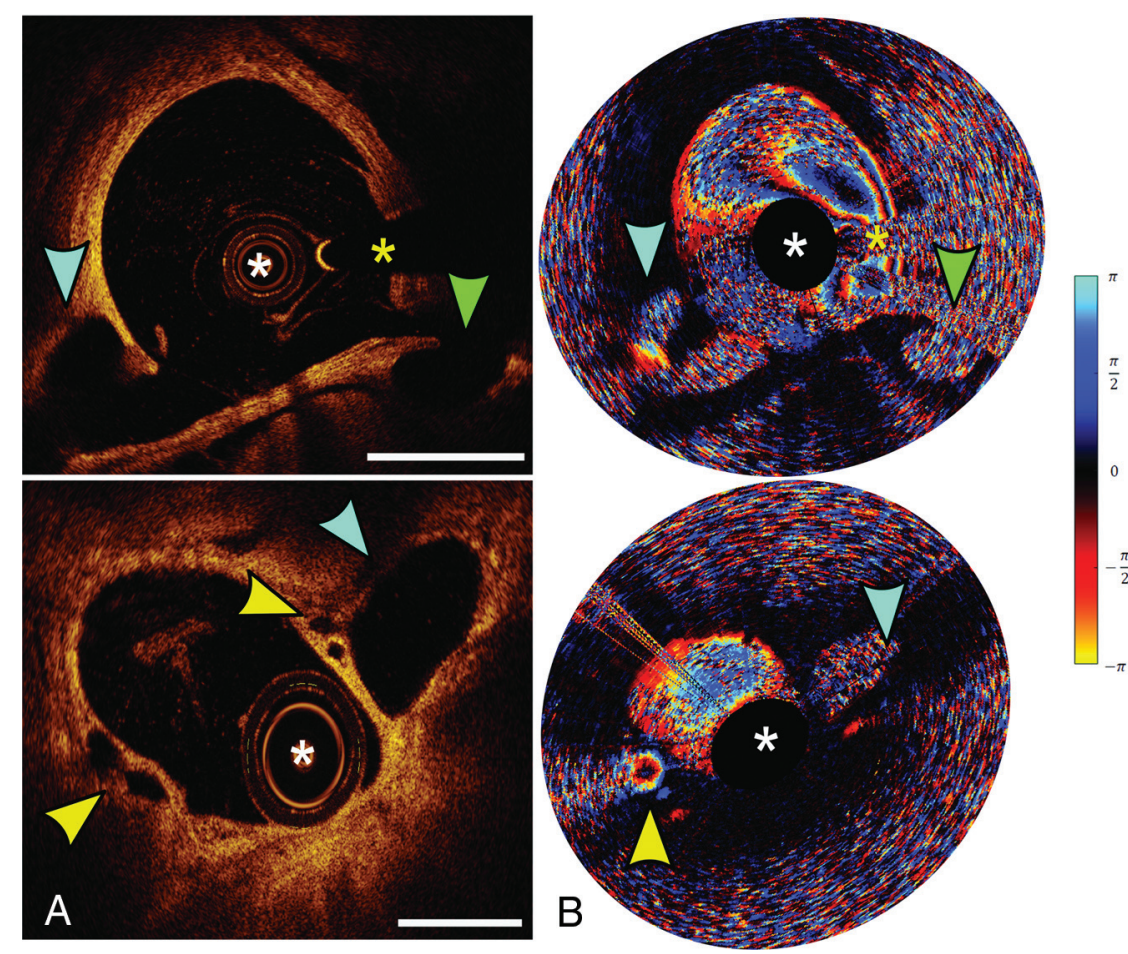

FIG 3. Corresponding structural and Doppler OCT imaging. A, Two separate structural images reveal large draining cortical veins (light blue and green arrows) along with dural arteries (yellow arrows) and their corresponding Doppler imaging with a phase-shift color map demonstrating flow in the sinus and dural arteries (B). The white asterisk is the OCT lens, and the yellow asterisk is the artifact from the wire. White bars $=2 \mathrm{~mm}$.
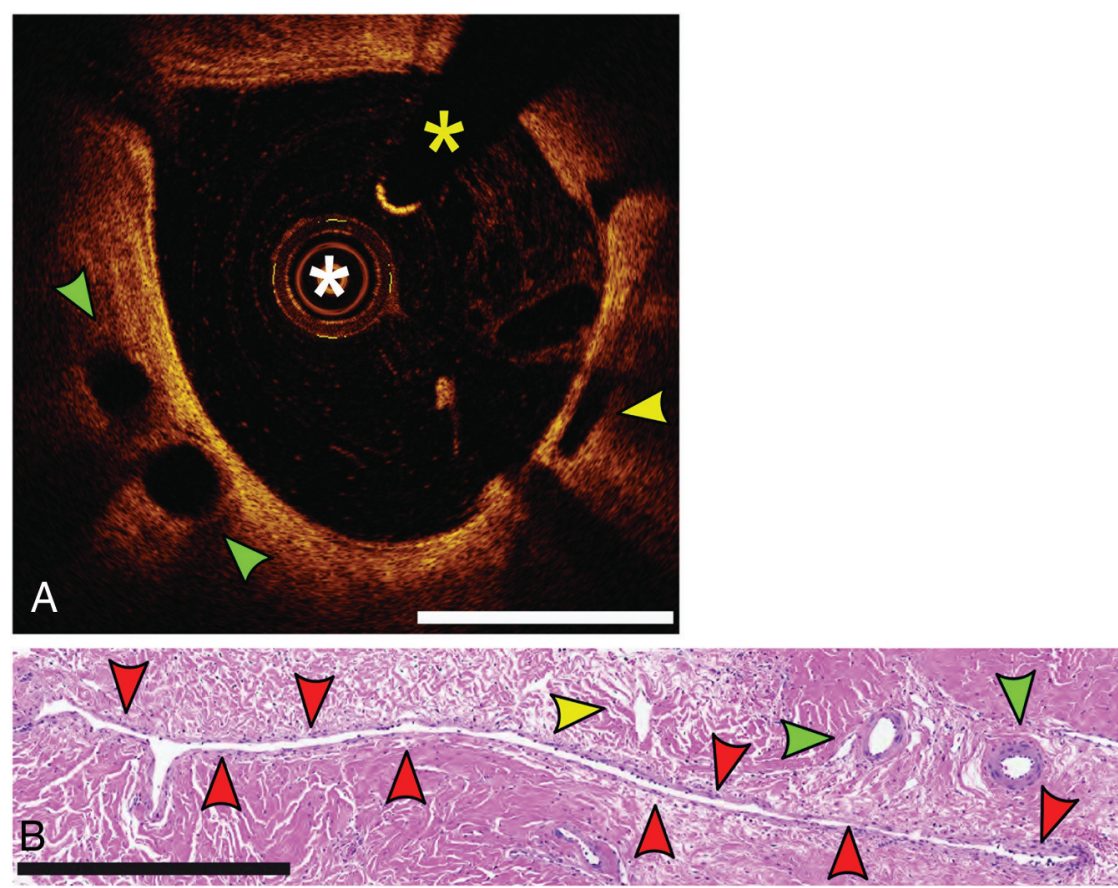

FIG 4. OCT imaging and corresponding histologic cross-section. A, OCT shows 2 dural arteries (green arrows) and a dural venule (yellow arrow). B. Histologic cross-section with H\&E staining. The sinus is collapsed, and the lumen is outlined with red arrows, along with adjacent arterioles (green arrows) and a venule (yellow arrow). The white asterisk is the OCT lens, and the yellow asterisk is the artifact from the wire. White bars $=2 \mathrm{~mm}$. Black scale bar $=500 \mu \mathrm{m}$. common femoral artery, and another, into the right femoral vein. First, a 6 F Envoy guide catheter (Codman \& Shurtleff) was used in conjunction with a $150-\mathrm{cm}$ Glidewire (Terumo) to select the right ascending pharyngeal artery. The guide catheter was positioned before the rete mirabile. The C-Arm (Philips Healthcare) was then positioned in a true lateral view, and cerebral angiography was performed to visualize the sinus.

Next, a second 6F Envoy was positioned in the right internal jugular vein. Combined arterial pump injections from the ascending pharyngeal artery and venous hand injection, timed to the late venous phase of the arterial pump injection, were performed to visualize the internal jugular and sigmoid junction (Fig 1A). A Transend microguidewire (Stryker) was navigated into the superior sagittal sinus via the sigmoid and transverse sinuses under a roadmap technique.

\section{Optical Coherence Tomography Imaging}

The Dragonfly OCT catheter (Abbott) was used for image acquisition. The following steps were sequentially followed for image acquisition: 1) Load an automated injection pump with 150-mL mixture of 50:50 contrast and saline. The pump is connected to the Envoy catheter in the ascending pharyngeal artery. This is used to clear the blood within the sinus lumen during OCT image acquisition. 2) Mount the OCT catheter on the Transend microguidewire and advance the device in a monorail fashion into the superior sagittal sinus, 3) position the OCT catheter so that the optical lens radiopaque marker is in the anterior third of the sinus (Fig $1 B), 4$ ) inject $3 \mathrm{~mL}$ per second for 8 seconds total ( $24 \mathrm{~mL}$ total) via the pump, and 5) enable manual OCT pullback mode and initiate the pullback manually once the lumen begins to clear. The OCT catheter performs the motorized automated pullback of $54 \mathrm{~mm}$ total. The OCT imaging frequency is 100 frames per second, with a total of 540 cross-sectional images generated per pullback. 


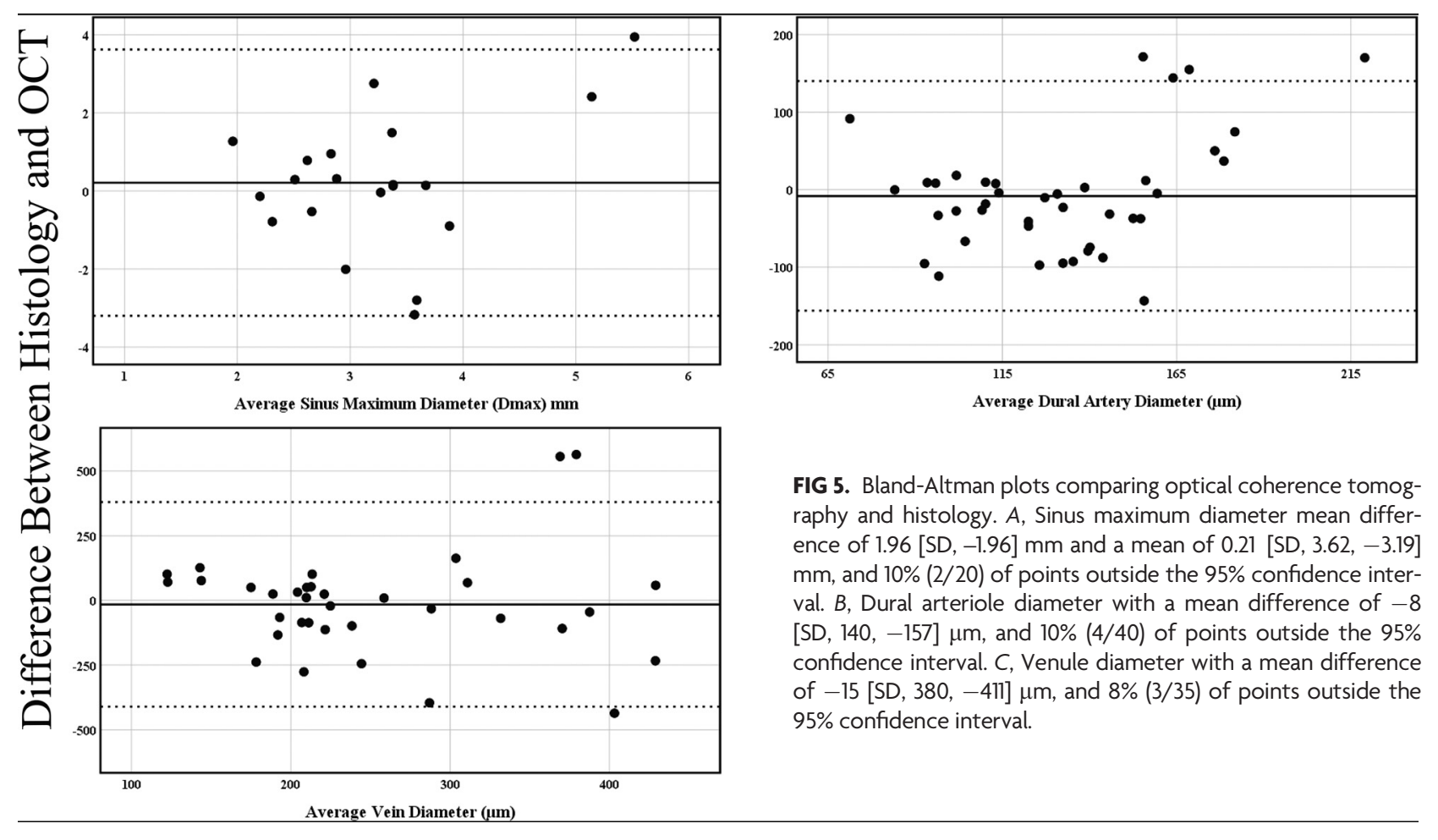

The technique for Doppler OCT (dOCT) image acquisition has been previously described by Vuong et al. ${ }^{9}$ Briefly, a split spectrum dOCT technique was used to reduce phase noise without incorporating external bulk optical devices. During the image acquisition, an arterial injection of $2 \mathrm{~mL}$ per second for 10 seconds (20 $\mathrm{mL}$ total) was performed. Both OCT and dOCT images were reviewed independently by 2 physicians with extensive experience in analyzing OCT images (C.R.P. and V.X.D.Y.).

\section{Pathology}

After OCT imaging and the sacrifice of the animal, craniotomy was performed as well as resection of the superior sagittal sinus beyond the torcula into the bilateral transverse sinuses, with 2-cm margins of the adjacent dura along with preservation of draining cortical veins. The resected specimen segments were fixed in $10 \%$ neutral buffered formalin. Then the sinuses were sectioned coronally into 5$\mathrm{mm}$ segments and submitted in total from anterior to posterior in tissue blocks for embedding in paraffin. One level through each of these tissue blocks was created, and 5 - $\mu$ m-thick tissue sections were mounted on glass slides and stained with H\&E. H\&E-stained slides from each block of the sampled sinus were scanned by an Aperio AT Turbo slide scanner (Leica Biosystems), and the resulting digital histology images were examined using the Aperio eSlide Manager (https://www.leicabiosystems.com/digital-pathology/manage/ aperio-eslide-manager/) by an experienced neuropathologist (J.K.).

For each digital histology image, the maximum diameter of the observed lumen of the superior sagittal sinus and/or transverse sinuses was measured digitally in micrometers, and the location and luminal diameter of the adjacent arterioles and venules were also recorded in micrometers. Digital photomicrographs were obtained, with and without the annotated dimensions, and the digital photomicrographs were compared with the images obtained by structural OCT. For statistical analysis, Bland-Altman plots were generated using the mean difference between histology and OCT scores and a $1.96 \mathrm{SD}$ with respect to the various luminal characteristics.

\section{RESULTS}

Cerebral venous sinus access was successful in all animals, and successful OCT and dOCT images were acquired in 3 of 4 swine. In swine 2, access was gained into the superior sagittal sinus with the 0.014-inch microwire; however, the OCT catheter could not traverse a suspected venous valve at the junction of the internal jugular vein and the sigmoid sinus; therefore, no OCT or dOCT images were obtained. In the remaining 3 swine, technically successful images were acquired. For image acquisitions to be defined as successful, they had to meet all the following criteria: 1) navigation of the OCT catheter to the appropriate location within the sinus, 2) clearing of luminal blood with minimal artifacts from red blood cells, 3) capturing circumferential OCT images of the entire sinus lumen along the entire region of interest, 4) identifying normal anatomic structures when present, and 5) identifying luminal lesions and vessel wall lesions when present.

The luminal environment could be characterized without ambiguity (Fig 2). The luminal diameter could readily be calculated, along with visualization of dural arteries and draining cortical veins (Fig 3). When present, luminal thrombus, likely secondary to microguidewire manipulation, could be observed and characterized as red or white thrombus.

The average maximum diameter of the sinus was $3.14 \mathrm{~mm}$ (95\% CI, 5.11-1.17 mm). The average diameter of adjacent dural arteries was $135 \mu \mathrm{m}(95 \% \mathrm{CI}, 211-60 \mu \mathrm{m})$. The average venule diameter was $260 \mu \mathrm{m}(95 \% \mathrm{CI}, 520-1 \mu \mathrm{m})$. Bland-Altman analysis 

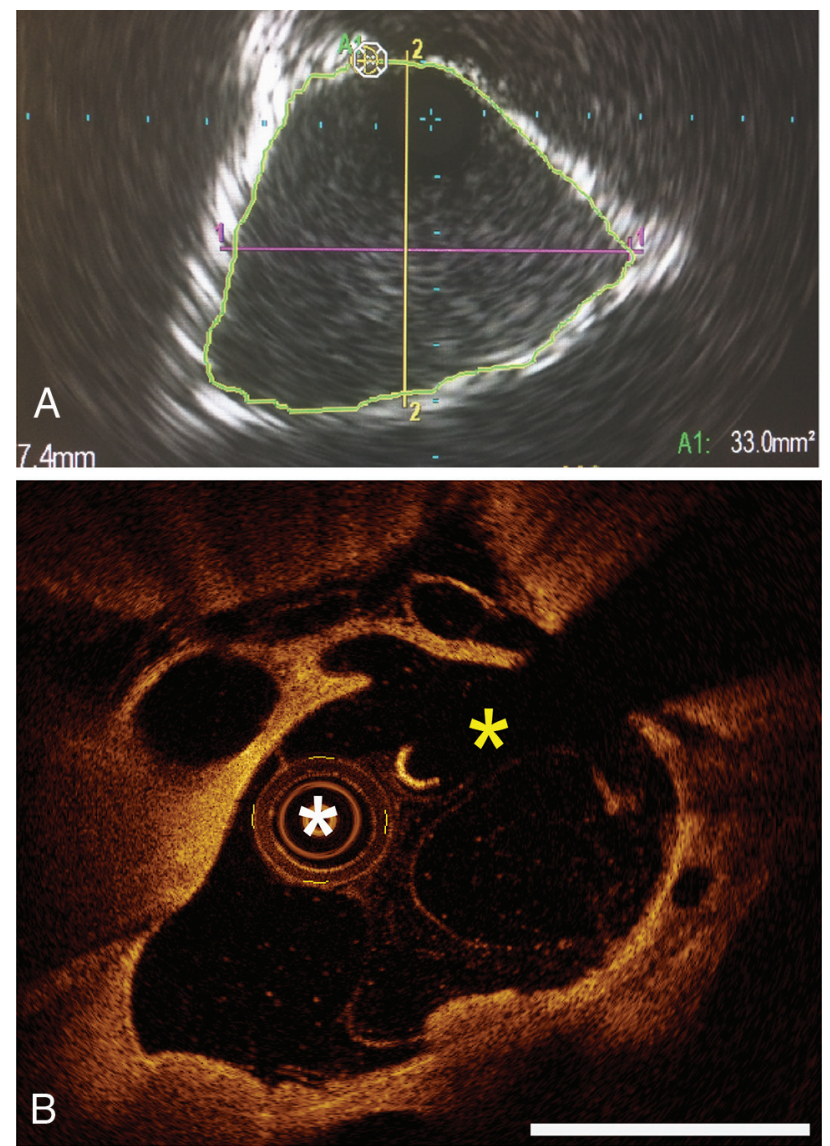

FIG 6. IVUS and OCT. A, IVUS of human superior sagittal sinus to determine the maximal diameter and circumference $(A)$, and swine structural OCT imaging of the superior sagittal sinus $(B)$. Although direct anatomic comparison cannot be made, OCT can clearly provide improved visualization of the sinus lumen and adjacent vessels. The white asterisk is the OCT lens, and the yellow asterisk is the artifact from the wire. White bars $=2 \mathrm{~mm}$. Adapted with permission from Boddu et al. ${ }^{5}$

demonstrated good agreement between histology (Fig 4) and OCT images across Dmax and arterial/venule diameter measurements (Fig 5). For Dmax, a mean difference with 1.96 and $-1.96 \mathrm{SD}$ (mean, [SD]) of $0.21 \mathrm{~mm}[3.62,-3.19]$ was observed, with $10 \%(2 / 20)$ of points outside the $95 \%$ confidence interval (Figure 5). For dural arteriole diameter, a mean difference of $-8 \mu \mathrm{m}[140,-157]$ was observed, with $10 \%(4 / 40)$ of points outside the $95 \%$ confidence interval (Fig 5). For venule diameter, a mean difference of $-15 \mu \mathrm{m}$ $[380,-411]$ was observed, with $8 \%(3 / 35)$ of points outside the 95\% confidence interval (Fig 5).

\section{DISCUSSION}

The goal of this study was to test the hypothesis that endovascular optical coherence tomography can characterize the cerebral venous sinus luminal environment and visualize draining cortical veins and dural arteries with near-histologic accuracy. In this preclinical swine study, OCT imaging was found to be technically feasible, with luminal blood clearing through a single arterial injection, and accurate compared with histology findings.

The first described use of intravascular imaging of the cerebral venous sinuses was by Tsumoto et $\mathrm{al}^{10}{ }^{1}$ in 2003 . They described IVUS imaging in a patient with sigmoid sinus

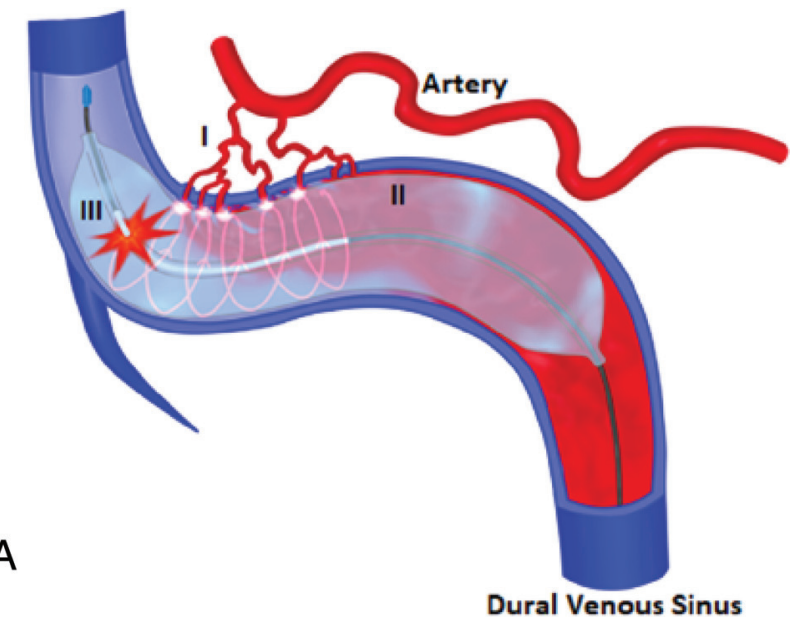

Dural Venous Sinus

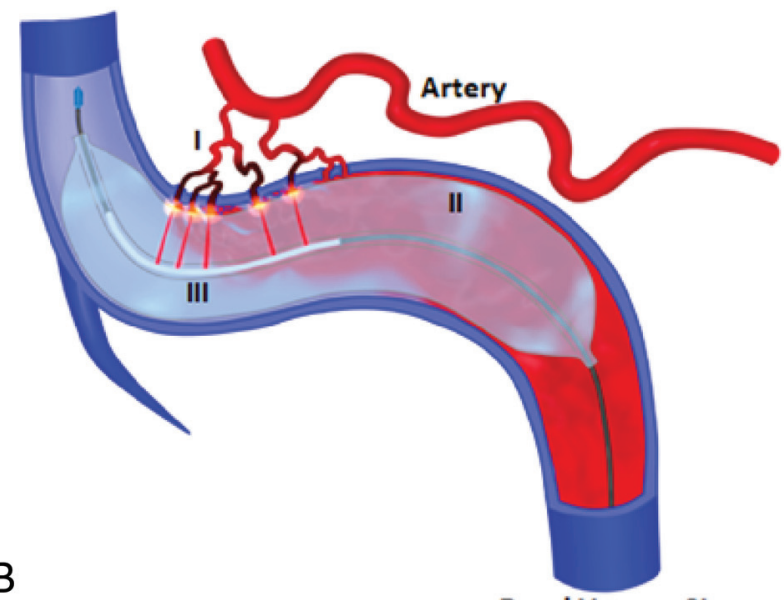

Dural Venous Sinus

FIG 7. A dural arteriovenous fistula imaged and treated using novel OCT technology. A, The OCT device is navigated into the dural venous sinus and through a transparent dual-lumen balloon (II), optical imaging is undertaken (III) to identify the exact spatial position of arterial feeders (I) into the dural venous sinus. $B$, Laser ablation of the arterial feeders is accomplished under image guidance through the saline-filled balloon. The arterial feeders (I) previously identified now undergo image-guided laser ablation (III) through the transparent dual lumen balloon (II). Adapted from Pasarikovski et al. ${ }^{21}$

restenosis after stent placement for venous hypertension and identified intimal proliferation over the stent causing the stenosis. Radvany et al $^{11}$ demonstrated, for the first time using IVUS, that transverse sinus stenosis in 2 patients with IIH was the result of intraluminal abnormalities (echogenic material within the sinus) and not extrinsic compression from raised intracranial pressure. Several authors have since described the use of IVUS as a diagnostic tool and treatment adjunct for patients with IIH, particularly highlighting the inability of CTV/MRV or even DSA to differentiate thrombus and structural stenosis and to select proper stent sizes. ${ }^{12-14}$

To our knowledge, cerebral venous sinus imaging with OCT has not been previously reported in humans or in preclinical animal studies. Gounis et al, ${ }^{15}$ in 2018, described the use of optical coherence tomography in neurointerventional surgery. Current arterial applications of OCT include the following: visualizing stent-vessel interactions during carotid stent placement and flow diversion for aneurysmal embolization, characterizing intracranial 
atherosclerotic disease, and after endovascular thrombectomy, assessing residual thrombus. ${ }^{16-20}$

Optical coherence tomography has approximately 10 times the spatial resolution of IVUS, producing much higher quality images and enabling improved visualization of the lumen, venous sinus wall, draining cortical veins, and dural arteries (Fig 6). This improved visualization has great potential with respect to aiding in the diagnosis and treatment of patients with cerebral venous pathology. Abnormalities such as sinus septations and luminal thrombus can be visualized readily. The potential applications of endovascular OCT in treating diseases of the CVS is boundless. For example, it is hypothesized that the near-histologic accuracy of OCT in detecting and characterizing dural arteries could be used for image-guided ablation of arterial feeders in a dural arteriovenous fistula (Fig 7).

In this swine model, clearing blood within the sinus lumen for OCT acquisition was achieved with an arterial injection. The authors could not place a large-gauge catheter $(\geq 5 \mathrm{~F})$ in the swine sinus (distal internal jugular or sigmoid) to try to clear luminal blood directly because the swine sinus diameter is small. On the other hand, it is likely that in human imaging, the sinus blood can be cleared with an intravenous injection through a guide catheter positioned in the sinus.

There are several limitations to this study. First, OCT was tested in swine, whereas human CVS are larger with the possibility of increased tortuosity; therefore, difficult navigation of the OCT catheter is possible in certain patients. Second, the safety of OCT in human imaging needs to be further studied. Although the OCT catheter is delivered via a monorail technique over a 0.014 -inch wire, venous perforation is possible via the stiff catheter tip.

\section{CONCLUSIONS}

Endovascular optical coherence tomography imaging was technically feasible in this preclinical swine study. Adoption of this imaging technique in the human cerebral venous sinus could aid in the diagnosis, treatment, and understanding of the pathophysiology of dural arteriovenous fistulas, cerebral venous sinus thrombosis, and IIH. Human safety and feasibility studies are needed.

Disclosures: Joel Ramjist-RELATED: Grant: Canadian Foundation for Innovation, Comments: The grant paid for the establishment of biophotonics research equipment in neurosurgical practice, grant title: "Biophotonics Experimental Operating Room."* Yuta Dobashi-UNRELATED: Employment: University of Toronto. *Money paid to the institution.

\section{REFERENCES}

1. Mack J, Squier W, Eastman JT. Anatomy and development of the meninges: implications for subdural collections and CSF circulation. Pediatr Radiol 2009;39:200-10 CrossRef Medline

2. Vignes JR, Dagain A, Guerin J, et al. A hypothesis of cerebral venous system regulation based on a study of the junction between the cortical bridging veins and the superior sagittal sinus: laboratory investigation. J Neurosurg 2007;107:1205-10 CrossRef Medline

3. Adeeb N, Mortazavi MM, Tubbs RS, et al. The cranial dura mater: a review of its history, embryology, and anatomy. Childs Nerv Syst 2012;28:827-37 CrossRef Medline

4. Saposnik G, Barinagarrementeria F, Brown RD Jr, et al; American Heart Association Stroke Council and the Council on Epidemiology and Prevention. Diagnosis and management of cerebral venous thrombosis: a statement for healthcare professionals from the American Heart Association/American Stroke Association. Stroke 2011;42:1158-92 CrossRef Medline

5. Boddu SR, Gobin P, Oliveira C, et al. Anatomic measurements of cerebral venous sinuses in idiopathic intracranial hypertension patients. PLoS One 2018;13:e0196275 CrossRef Medline

6. Karmon Y, Zivadinov R, Weinstock-Guttman B, et al. Comparison of intravascular ultrasound with conventional venography for detection of extracranial venous abnormalities indicative of chronic cerebrospinal venous insufficiency. J Vasc Interv Radiol 2013;24:1487-98 CrossRef Medline

7. Tearney GJ, Regar E, Akasaka T, et al; International Working Group for Intravascular Optical Coherence Tomography (IWGIVOCT). Consensus standards for acquisition, measurement, and reporting of intravascular optical coherence tomography studies: a report from the International Working Group for Intravascular Optical Coherence Tomography Standardization and Validation. J Am Coll Cardiol 2012;59:1058-72 CrossRef Medline

8. Tearney GJ, Brezinski ME, Bouma BE, et al. In vivo endoscopic optical biopsy with optical coherence tomography. Science 1997;276:2037-39 CrossRef Medline

9. Vuong B, Genis H, Wong R, et al. Evaluation of flow velocities after carotid artery stenting through split spectrum Doppler optical coherence tomography and computational fluid dynamics modeling. Biomed Opt Express 2014;5:4405-16 CrossRef Medline

10. Tsumoto T, Miyamoto T, Shimizu M, et al. Restenosis of the sigmoid sinus after stenting for treatment of intracranial venous hypertension: case report. Neuroradiology 2003;45:911-15 CrossRef Medline

11. Radvany MG, Gomez J, Gailloud P. Intravascular ultrasound of the transverse sinus in two patients with pseudotumor cerebri: technical note. J Neurointerv Surg 2011;3:379-82 CrossRef Medline

12. Mokin M, Kan P, Abla AA, et al. Intravascular ultrasound in the evaluation and management of cerebral venous disease. World Neurosurg 2013;80:55.e7-13 CrossRef Medline

13. Buell TJ, Raper DM, Pomeraniec IJ, et al. Transient resolution of venous sinus stenosis after high-volume lumbar puncture in a patient with idiopathic intracranial hypertension. J Neurosurg 2018;129:15356 CrossRef Medline

14. Yan F, Rajah G, Ding Y, et al. Safety and efficacy of intravascular ultrasound as an adjunct to stenting for cerebral venous sinus stenosis-induced idiopathic intracranial hypertension: a pilot study. J Neurosurg 2019 Mar. [Epub ahead of print] CrossRef Medline

15. Gounis MJ, Ughi GJ, Marosfoi M, et al. Intravascular optical coherence tomography for neurointerventional surgery. Stroke $2018 \mathrm{Nov}$ 29. [Epub ahead of print] CrossRef Medline

16. Griessenauer CJ, Gupta R, Shi S, et al. Collar sign in incompletely occluded aneurysms after Pipeline embolization: evaluation with angiography and optical coherence tomography. AJNR Am J Neuroradiol 2017;38:323-26 CrossRef Medline

17. Dohad S, Zhu A, Krishnan S, et al. Optical coherence tomography guided carotid artery stent procedure: technique and potential applications. Catheter Cardiovasc Interv. 2018;91:521-30 CrossRef Medline

18. Pasarikovski CR, da Costa L, Pereira VM, et al. Optical coherence tomography-guided flow diversion for aneurysmal treatment. Neurol Clin Pract 2020;10:e30-32 CrossRef Medline

19. Pasarikovski CR, Ramjist J, da Costa L, et al. Optical coherence tomography imaging after endovascular thrombectomy for basilar artery occlusion: report of 3 cases. J Neurosurg 2020;133:1141-46 CrossRef Medline

20. Xu X, Li M, Liu R, et al. Optical coherence tomography evaluation of vertebrobasilar artery stenosis: case series and literature review. J Neurointerv Surg 2020;12:809-13 CrossRef Medline

21. Pasarikovski CR, Cardinell J, Yang VX. Perspective review on applications of optics in cerebral endovascular neurosurgery. J Biomed Opt 2019;24:1-7 CrossRef Medline 